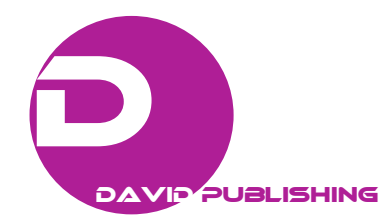

\title{
Behavior of a Nonlinear Difference Equation
}

$$
x_{n+1}=\frac{1-x_{n}}{A+\sum_{i=1}^{k} x_{n-i}}
$$

Mehmet Emre Erdogan ${ }^{1}$ and Kemal Uslu ${ }^{2}$

1. Huglu Vocational High School, Selcuk University, Konya, Turkey

2. Department of Science, Selcuk University, Konya, Turkey

Abstract: In this paper, we investigate the global behavior of the difference equation

$$
\begin{gathered}
x_{n+1}=\frac{1-x_{n}}{A+\sum_{i=1}^{k} x_{n-i}} \\
n=0,1, \ldots
\end{gathered}
$$

with the $A \in(-\infty,-1)$ is a real number, $k$ is a positive integer and the initial conditions $x_{-k}, \ldots, x_{0} \in(-\infty, 0]$.

Key words: Recursive sequences, global asymptotic stability, period-two solution, invariant interval.

\section{Introduction}

Recently, many researchers are interested in the boundedness, invariant intervals, periodic character and global asymptotic stability of all positive solutions of the nonlinear difference equations, see [1, 2, 4-7, 10-12]. In particular He et al. [3], Li and Sun [9] and Yan et al. [13] investigated the global asymptotic stability of all positive solutions of the following rational recursive sequences

$$
\begin{gathered}
x_{n+1}=\frac{\alpha-b x_{n-k}}{A-x_{n}}, n=0,1, \ldots \\
x_{n+1}=\frac{\alpha-\beta x_{n}}{\gamma+x_{n-k}}, n=0,1, \ldots \\
x_{n+1}=\frac{\alpha-\beta x_{n}}{\gamma-x_{n-k}}, n=0,1, \ldots
\end{gathered}
$$

respectively, where the coefficients $\alpha, \beta, \gamma, A, b$ are nonnegative real numbers and $k \in\{1,2, \ldots\}$, and showed that every positive equilibruim of these equations is a global attractor with a basin that

Corresponding Author: Mehmet Emre Erdogan, Ph.D., research fields: stability, oscillation andmathematical modelling. depends on certain conditions imposed on the coefficients. Furthermore, they obtained sufficient conditions for the globally asymptotically stable of the positive equilibria of these equations.

In 2004, Li et al. [8] studied the difference equation

$$
x_{n+1}=\frac{\alpha+\beta x_{n}}{A+x_{n-k}}, n=0,1, \ldots
$$

Where $\alpha, \beta, A \in(0, \infty), k$ is a positive integer and the initial conditions $x_{-k}, \ldots, x_{0}$ are arbitrary positive numbers. They investigated the global asymptotic stability of all positive solutions and obtained the global attractivity of the unique positive equilibrium of the equation.

It is also noted that the above mentioned references [2, 4-7, 10-12] only considered the global attractivity of positive solutions of difference equation. They did notfurther provide the global attractivity of negative solutions of difference equation. In this paper, we will deal with the global attractivity of negative solutions for the following difference equation

$$
x_{n+1}=\frac{1-x_{n}}{A+\sum_{i=1}^{k} x_{n-i}}, n=0,1, \ldots
$$

with the $A \in(-\infty,-1)$ is a real number, $k$ is a 
positive integer and the initial conditions $x_{-k}, \ldots, x_{0} \in$ $(-\infty, 0]$. Our aim is to study the periodic character, invariant intervals and the global attractivity of all negative solutions of Eq. (1.1) is a global attractor with a basin that depends on certain conditions of the coefficient.

For the sake of convenience, we firstly present some definitions and known results which will be useful in the sequel. Let $I$ be some interval of real numbers $F$ be continuous function defined on $I^{k+1}$. Then, for initial conditions $x_{-k}, \ldots, x_{0} \in I$, it is easy to see that the difference equation

$$
x_{n+1}=F\left(x_{n}, x_{n-1}, \ldots, x_{n-k}\right), n=0,1, \ldots
$$

has a unique solution $\left\{x_{n}\right\}_{n=-k}^{\infty}$.

A point $\bar{x}$ is called an equilibrium of Eq. (1.2) if

$$
\bar{x}=F(\bar{x}, \ldots, \bar{x}) .
$$

That is, $x_{n}=\bar{x}$ for $n \geq 0$ is a solution of Eq. (1.2), or equivalently, $\bar{x}$ is a fixed point of $F$.

An interval $J \subseteq I$ is called an invariant interval for Eq. (1.2) if

$$
x_{-k}, \ldots, x_{0} \in J \Rightarrow x_{n} \in J \text { for all } n>0 .
$$

That is, every solution of Eq. (1.2) with initial conditions in $j$ remains in $j$.

The linearized equation associated with Eq. (1.2) about the equilibruim $\bar{x}$ is

$$
y_{n+1}=\sum_{i=0}^{k} \frac{\partial F}{\partial u_{i}}(\bar{x}, \ldots, \bar{x}) y_{n-i}, n=0,1, \ldots
$$

and its characteristic equation is

$$
\lambda^{k+1}=\sum_{i=0}^{k} \frac{\partial F}{\partial u_{i}}(\bar{x}, \ldots, \bar{x}) \lambda^{k-i} .
$$

Definition 1. Let $\bar{x}$ be an equilibrium point of Eq. (1.2).

(i) The equilibrium $\bar{x}$ is called locally stable if for every $\varepsilon>0$, there exists $\delta>0$ such that if $x_{0}, \ldots, x_{-k} \in I$ and $\left|x_{0}-x\right|+\cdots+\left|x_{-k}-x\right|<\delta$, then $\left|x_{n}-x\right|<\varepsilon$, for all $n \geq-k$.

(ii) The equilibrium $\bar{x}$ is called locally asymptotically stable if it is locally stable and if there exists $\gamma>0$ such that if $x_{0}, \ldots, x_{-k} \in I$ and $\left|x_{0}-x\right|+\cdots+\left|x_{-k}-x\right|<\gamma$, then $\lim _{n \rightarrow \infty} x_{n}=\bar{x}$. (iii) The equilibrium $\bar{x}$ is called global attractor if for every $x_{0}, \ldots, x_{-k} \in I$, we have $\lim _{n \rightarrow \infty} x_{n}=\bar{x}$.

(iv) The equilibrium $\bar{x}$ is called globally asymptotically stable if it is locally stable and is a global attractor.

(v) The equilibrium $\bar{x}$ is called unstable if it is not stable.

The following two results will be useful in establishing the locally asymptotically stable and global attractivity character of the equilibrium of Eq. (1.1).

Theorem 1. A necessary and sufficient condition for all roots of the cubic equation with real coefficients

$$
\lambda^{3}+a_{2} \lambda^{2}+a_{1} \lambda+\lambda^{3} a_{0}=0
$$

to lie in the open disc $|\lambda|<1$ is

$$
\begin{gathered}
\left|a_{2}+a_{0}\right|<1+a_{1},\left|a_{2}-3 a_{0}\right|<3-a_{1} \text { and }_{0}{ }^{2}+ \\
a_{1}-a_{0} a_{2}<1 .
\end{gathered}
$$

Theorem 2. Let $[a, b]$ be an interval of real numbers, and assume that

$$
g:[a, b]^{3} \rightarrow[a, b]
$$

is a continuous function satisfying the following properties:

(a) $g(u, v, w)$ is non-increasing in $u$ and $w$, and is non-decreasing in $v$.

(b) If $(m, M) \in[a, b] \times[a, b]$ is a solution of the system

$$
m=g(M, m, M) \text { and } M=g(m, M, m)
$$

then $m=M$.

Then the difference equation

$$
x_{n+1}=g\left(x_{n}, x_{n-1}, x_{n-2}\right), n=0,1, \ldots \text { (1.5) }
$$

has a unique equilibrium $\bar{x} \in[a, b]$, and every solution of Eq. (1.5) converges to $\bar{x}$.

\section{Main Results}

In this section, we investigate the locally asymptotically stable, period-two solutions, invariant intervals and global attractivity of all negative solutions of Eq. (1.1) under the assumptions that $A \in(-\infty,-1)$ is a real number, $k$ is a positive integer and the initial conditions are non-positive. 
The unique negative equilibrium of Eq. (1.1) is

$$
x=\frac{-(A+1)-\sqrt{(A+1)^{2}+4 k}}{2 k}
$$

The linearized equation of Eq. (1.1) about the equilibrium $\bar{x}$ is

$$
\begin{aligned}
& y_{n+1}+\frac{1}{A+k \bar{x}} y_{n}+\frac{\bar{x}}{A+k \bar{x}} y_{n-1}+\cdots \\
& +\frac{\bar{x}}{A+k \bar{x}} y_{n-k}=0, n=0,1, \ldots
\end{aligned}
$$

and its characteristic equation is

$$
\lambda^{k+1}+\frac{1}{(A+k x) \lambda^{k}}+\frac{x}{(A+k x) \lambda^{k-1}}+\ldots+\frac{x}{A+k x}=0
$$

By Definition 1 and Theorem 2, we have the following result.

Theorem 3. Assume $A<0$. Then the unique negative equilibrium $\bar{x}=\frac{-(A+1)-\sqrt{(A+1)^{2}+4 k}}{2 k}$ of Eq. (1.1) is locally asymptotically stable.

Theorem 4. Eq. (1.1) has no negative solution with prime period-two.

Proof: Assume for the sake of contradiction that there exist distinctive negative real numbers $\phi$ and $\psi$, such that

$$
\ldots, \phi, \psi, \phi, \psi, \ldots
$$

is a prime period-two solution of Eq. (1.1). Then, there are two cases to be considered.

Case (i): If $k$ is odd, in this case $x_{n+1}=x_{n-k}, \phi$ and $\psi$ satisfy the system

$$
\phi=\frac{1-\psi}{A+\phi+\psi+\cdots \phi}, \quad \psi=\frac{1-\phi}{A+\psi+\phi+\cdots+\psi}
$$

then we have

$$
(\phi-\psi)\left(A+\frac{k+1}{2}(\phi+\psi)-1\right)=0 .
$$

In view of $A+\frac{k+1}{2}(\phi+\psi)-1<0$, then $\phi=\psi$.

This contradicts the hypothesis that $\phi \neq \psi$.

Case (ii): If $k$ is even, in this case $x_{n+1}=x_{n-k}$, $\phi$ and $\psi$ satisfy the system

$$
\phi=\frac{1-\psi}{A+\phi+\psi+\cdots+\psi}, \quad \psi=\frac{1-\phi}{A+\psi+\phi+\cdots+\phi}
$$

then we have

$$
(\phi-\psi)\left(A+\frac{k}{2}(\phi+\psi)-1\right)=0 .
$$

Clearly, $\quad A+\frac{k}{2}(\phi+\psi)-1<0$, so $\phi=\psi$, which is also a contradiction. The proof is complete.

Let

$$
f(u, v, \ldots)=\frac{1-u}{A+v+\ldots}
$$

Lemma-1. Assume $A<0$. Then the following statements are true.

(i) $-1<x=\frac{-(A+1)-\sqrt{(A+1)^{2}+4 k}}{2 k}<0$.

(ii) If $u, v, w \in(-\infty, 0]$, then $f(u, v, w)$ is a strictly increasing function in $u$, and a strictly decreasing function in $v$ and $w$.

The proof is simple and omitted.

Lemma-2. Assume that $A \in(-\infty,-1]$ and let $\left\{x_{n}\right\}_{n=-k}^{\infty}$ be a solution of Eq. (1.1). If $x_{i} \in(-\infty, 0]$ for $i=-k,-k+1, \ldots,-1$ and $x_{0} \in[A, 0]$, then $A \leq x_{n}<0$ for $n=1,2, \ldots$

Proof:

$$
\begin{aligned}
& A \leq \frac{1-A}{A} \leq \frac{1-A}{A+x_{-k}+x_{-k+1}} \leq x_{1} \\
& =\frac{1-x_{0}}{A+x_{-k}+x_{-k+1}} \\
& \leq \frac{1}{A+x_{-k}+x_{-k+1}}<0
\end{aligned}
$$

and

$$
\begin{aligned}
& A \leq \frac{1-A}{A} \leq \frac{1-A}{A+x_{-k+1}+x_{-k+2}} \leq x_{1} \\
& =\frac{1-x_{0}}{A+x_{-k+1}+x_{-k+2}} \\
& \leq \frac{1}{A+x_{-k+1}+x_{-k+2}}<0
\end{aligned}
$$

The result now follows by induction. The proof is complete.

Theorem 5. Assume that $A \in(-\infty,-1]$. Then $[A, 0]$ is an invariant interval of Eq. (1.1).

\section{Proof:}

Let $\left\{x_{n}\right\}_{n=-k}^{\infty}$ be a solution of Eq. (1.1) with initial conditions $x_{-k}, \ldots, x_{-1}, x_{0} \in[A, 0]$. By Lemma-1, the 
function $f(u, v, w)$ is strictly increasing in $u$, and strictly decreasing in $v$ and $w$ for each fixed $u, v, w \in(-\infty, 0]$, we have

$$
x_{1}=f\left(x_{0}, x_{-k}, x_{-k+1}\right)<f(0, A, A)=\frac{1}{3 A}<0
$$

And

$$
x_{1}=f\left(x_{0}, x_{-k}, x_{-k+1}\right)>f(A, 0,0)=\frac{1-A}{A} \geq A,
$$

which implies that $x_{1} \in[A, 0]$. By the induction, it follows that $x_{n} \in[A, 0]$ for $n \geq 1$. The proof is complete.

Theorem 6. Assume that $A \in(-\infty,-1]$. Then the unique negative equilibrium $\bar{x}$ of Eq. (1.1) is a global attractor with a basin

$$
S=[A, 0]^{k+1} .
$$

\section{Proof:}

Let $\left\{x_{n}\right\}_{n=-k}^{\infty}$ be a solution of Eq. (1.1) with initial conditions $x_{-k}, \ldots, x_{-1}, x_{0} \in S$. Clearly, the function $f(u, v, w)$ defined in Lemma-1 is continuous, and nondecreasing in $u$ and nonincreasing in $v$ and $w$ on the invariant interval $[A, 0]$. Let $m, M \in I$ be a solution of the system

$$
m=f(m, M, M), \quad M=f(M, m, m)
$$

then $(m-M)(1+A)=0$. Since $A+1<0$, we get $m=M$. By Theorem-3, we have $\lim _{n \rightarrow \infty} x_{n}=\bar{x}$, the proof is complete.

Theorem 7. Assume that $A \in(-\infty,-1]$. Then the unique negative equilibrium $\bar{x}$ of Eq. (1.1) is a global attractor with a basin

\section{Proof:}

$$
S=(-\infty, 0]^{k+1} \times[A, 0] .
$$

Let $\left\{x_{n}\right\}_{n=-k}^{\infty}$ be a solution of Eq. (1.1) with initial conditions $x_{-k}, \ldots, x_{-1}, x_{0} \in S$. Then by Lemma-2, we havex $_{n} \in[A, 0]$, for $n=1,2, \ldots$ By Theorem-6, we have $\lim _{n \rightarrow \infty} x_{n+k}=\bar{x}$, and so $\lim _{n \rightarrow \infty} x_{n}=\bar{x}$. The proof is complete.

\section{References}

[1] El-Owaidy, H. M., Ahmed, A. M., and Youssef, A. M. 2005. "The Dynamics of Recursive $x_{n+1}=\frac{\alpha x_{n-1}}{\beta+\gamma x_{n-2}^{p}}$." Applied Mathematics Letters: 1013-8.

[2] Amleh, A. M., Kirk, V., and Ladas, G. 2001. "On the
Dynamics of $x_{n+1}=\frac{a+b x_{n-1}}{A+B x_{n-2}}$, Math.” Sci. Res. Hot-Line 5: 1-15.

[3] Gibbons, C., Kulenovic, M., and Ladas, G. 2000. "On the Reqursive Sequence $y_{n+1}=\frac{\alpha+\beta y_{n-1}}{\gamma+y_{n}}$, Math.” Sci. Res. Hot-Line 4 (2): 1-11.

[4] Kocic, V. L., and Ladas, G. 1993. Global Behavior of Nonlinear Difference Equations of Higher Order with Applications, Kluwer Academic, Dordrecht.

[5] Kocic, V. L., Ladas, G., and Rodrigues, I. 1993. "On the Rational Recursive Sequences.” J.Math. Anal. Appl. 173: 127-57.

[6] El-Owaidy, H. M., Ahmed, A. M., and Mousa, M. S. 2003. "On the Recursive Sequences $x_{n+1}=\frac{-\alpha x_{n-1}}{\beta \pm x_{n}}$." $J$. Appl. Math. Comput. 145: 747-53.

[7] El-Owaidy, H. M., Ahmed, A. M., and Elsady, Z. 2004. "Global Attractivity of the Recursive Sequence $x_{n+1}=\frac{\alpha-\beta x_{n-1}}{\gamma+x_{n}}$.” J. Appl. Math. Comput. 151: 827-33.

[8] Stevic, S. 2006. "On the Recursive Sequence $x_{n+1}=$ $\frac{a x_{n-2 m+1}^{p}}{b+c x_{n-2 k}^{p-1}}$." J. Appl. Math. Comput. 21: 223-32.

[9] El-Owaidy, H. M., Ahmed, A. M., and Youssef, A. M. 2005. "On the Dynamics of $x_{n+1}=\frac{b x_{(n-1)}^{2}}{A+B x_{n-2}}$." Rostock Math Kollog. 59: 3-10.

[10] Hamza, A. E., and El-Sayed, M. A. 1998. "Stability Problem of Some Nonlinear Difference Equations.” Int. T. Math. and Math. Sci. 2: 331-40.

[11] Zhang, D. C., Shi, B., and Gai, M. T. 2001. "A Rational Recursive Sequence." Computers and Math. with Applications 41: 301-6.

[12] Chen, D., and Li, X. 2009. Dynamics for Nonlinear Difference Equation $x_{n+1}=\frac{\alpha x_{n-k}}{\beta+\gamma x_{n-l}^{p}}$ Advances in Difference Equations Article ID 235691.

[13] Camouzis, E., Chatterjee, E., and Ladas, G. 2007. “On the Dynamics of $x_{n+1}=\frac{\delta x_{n-2}+x_{n-3}}{A+x_{n-3}}$." Mathematical Analysis and Applications 331: 230-9.

[14] Erdogan, M. E., Cinar, C., and Yalcinkaya, I. 2011. “On the Dynamics of the Recursive Sentence $x_{n+1}=$ $\frac{\alpha x_{n-1}}{\beta+\gamma x_{(n-2)}^{2} x_{n-4}+\gamma x_{(n-2)} x_{(n-4)}^{2}}$." Comput. Math. Appl. 61: 533-7.

[15] Hamza, A. E., and Khalaf-Allah, R. 2007. "Global Behavior of Higher Order Difference Equation.” J. Math. Stat. 3 (1): 17-20.

[16] Hamza, A. E., and Khalaf-Allah, R. 2008. "On the Recursive Sequence $x_{n+1}=\frac{A \prod_{i=1}^{k} x_{n-2 i-1}}{B+C \prod_{i=1}^{k-1} x_{n-2 i}}$." Comput. Math. Appl. 56: 1726-31.

[17] El-Metwally, H. 2009. "Qualitative Properties of Some Higher Order Difference Equations.” Comput. Math. Appl. 
58: 686-92.

[18] Yalcinkaya, I., and Cinar, C. 2009. "On the Dynamics of

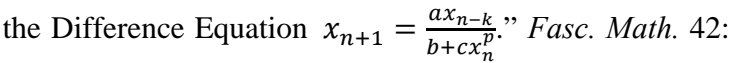
141-8.

[19] Dehghan, M., and Rastegar, N. 2009. "On the Global Behavior of a High-order Rational Difference Equation.” Comput. Phys. Comm. 180 (6): 873-8.
[20] Erdogan, M. E., and Cinar, C. 2013. "On the Dynamics of the $x_{n+1}=\frac{\alpha x_{n-1}}{\beta+\gamma \sum_{k=1}^{t} x_{n-2 k}^{p} \prod_{k=1}^{t} x_{n-2 k}^{q}}$." Fasc. Math. 50: 59-66.

[21] Kulenovic, M. R. S., and Merino, O. 2002. Discrete Dynamical Systems and Difference Equations with Mathematica, Chapman \& Hall/CRC Press, Boca Raton/London. 